\title{
REFLEXIONES EN TORNO A LA EMPRESARIALIDAD \\ Y EL DESARROLLO ECONÓMICO LOCAL
}

\author{
Guillermo Salvador Valdiviezo Ocampo \\ FACUltad DE Ciencias Sociales \\ UNIVERSIDAD AUTÓNOMA DE CHIAPAS
}

\begin{abstract}
RESUMEN
La empresarialidad es un tema que se ha abordado desde diferentes perspectivas teóricas, por eso en este artículo se analizan varios enfoques considerados importantes y se pone de manifiesto su relación con el análisis de la empresarialidad y el desarrollo económico local. También se analizan, desde la perspectiva del desarrollo económico local, algunas de las limitantes que registra uno de los modelos más utilizados para el estudio de la creación de empresas.
\end{abstract}

Palabras clave: empresarialidad, desarrollo local, instituciones, territorio. 


\begin{abstract}
Entrepreneurship is a topic that has been addressed from different theoretical perspectives. In this article the author analyzes some of the most significant approaches, and looks at the relationship between entrepreneurship and local economic development. He also uses a perspective of local economic development to analyze some of the limitations in one of the most frequently used models for studying the creation of businesses.
\end{abstract}

Key words: entrepreneurship, local development, institutions, territory. 


\section{INTRODUCCIÓN}

Diversos autores como Lucas (1990) y Hall y Jones (1999) reconocen que las disparidades a través de un país en términos de ingresos per cápita son muy persistentes, y que las diferencias en los aspectos tecnológicos son las que explican tales disparidades. Sin embargo, desde la perspectiva schumpeteriana los aspectos tecnológicos se asocian con los empresarios, ya que son ellos los principales determinantes de los resultados tecnológicos de un país porque los empresarios son los que adoptan nuevos métodos de producción, innovan en producto, y por lo tanto difunden el progreso tecnológico y hacen crecer la productividad. Bajo la misma lógica, Acs y Audretsch (2005) señalan que existe un estrecho vínculo entre la actividad empresarial y el mayor crecimiento registrado por algunas regiones.

Además de los argumentos teóricos, también se registran evidencias empíricas que indican que la empresarialidad contribuye al crecimiento económico, al aumento de la productividad, al rejuvenecimiento de los tejidos socioproductivos, a la innovación y a la generación de nuevos puestos de trabajo (Audretsch y Thurik 2001, OCDE 2001, Reynolds et al. 2002). La tasa de creación de empresas está asociada en forma positiva con el crecimiento económico, por ello un aumento de la actividad empresarial conlleva tasas de crecimiento económico más altas. En el ámbito local o regional es donde el impacto de la empresarialidad suele verse con mayor claridad. En las áreas geográficas con mayor natalidad de firmas y dinamismo empresarial el uso de los recursos económicos es más eficiente, y el crecimiento de la economía y las condiciones de vida de la población suelen ser superiores al de las áreas donde el índice de creación de empresas es más débil. La respuesta al porqué se mantienen a lo largo del tiempo las disparidades regionales, 
probablemente se pueda en parte encontrar en los bajos índices de empresarialidad de algunas regiones.

Para comprender los principales factores que contribuyen u obstaculizan la creación de empresas es conveniente revisar algunos de los componentes de los enfoques teóricos más relevantes. Por ello, en este trabajo se realiza solamente una revisión teórica de los principales enfoques en torno a la empresarialidad. En la primera sección del trabajo se comenta brevemente la concepción de empresa en el planteamiento neoclásico convencional ${ }^{1}$ la segunda toma en cuenta el enfoque neoinstitucionalista; en la siguiente el enfoque evolucionista; la cuarta sección presenta el enfoque del desarrollo económico local; le sigue el modelo Global Entrepreneurship Monitor, GEM, ${ }^{2}$ de creación de empresas, finalmente se presentan las conclusiones.

\section{ENFOQUE NEOCLÁSICO}

Según Coriat y Weinstein $(2002,2005)$, la visión de empresa en el marco del enfoque neoclásico ortodoxo basado en Walras —enfoque marginalista- se sustenta en tres aspectos: a) el objetivo del análisis es la búsqueda de condiciones de equilibrio en situaciones de competencia e información perfecta, ausencia de incertidumbre y un determinado estado de las técnicas; b) las firmas funcionan bajo un esquema de racionalidad perfecta, lo que les permite tomar siempre decisiones acertadas y buscar el objetivo de maximizar beneficios; c) se privilegia el análisis del intercambio más que el de los procesos de producción.

En este contexto, la empresa tiene como principal función aplicar los factores de la producción a los insumos con el objeto de producir bienes y servicios. Por ello, desde el punto de vista neoclásico tradicional, los únicos datos del «ambiente» que debe conocer son 
precios de productos y factores. Lo que supone que la empresa realiza esta tarea eficientemente en tanto conoce, y puede aplicar de manera adecuada, todas las técnicas disponibles. También se asume que hay una cooperación fluida y ausencia de conflictos entre todos aquellos que están involucrados en el funcionamiento de la empresa.

Derivado del enfoque neoclásico convencional de empresa, la actividad de los empresarios resulta irrelevante porque su participación se reduce a efectuar los cálculos que permiten maximizar beneficios a su empresa ante los cambios en los datos generados por el mercado. ${ }^{3}$ El enfoque neoclásico tradicional supone que la actividad de los empresarios está determinada por las condiciones que enfrentan. En otras palabras, solamente refleja condiciones objetivas de los sectores y mercados en los que operan. En consecuencia, ante un mismo cuadro exógeno, todas las firmas tenderán a comportarse de modo similar; de igual manera, si las condiciones de mercado cambian también lo hará, homogéneamente, el comportamiento de las empresas.

\section{ENFOQUE NEOINSTITUCIONALISTA}

Dentro de esta corriente se pueden encontrar algunas propuestas, como la de Coase (1960), que comparten ciertos postulados con la escuela neoclásica, pero a medida que se relajan los supuestos neoclásicos se van alejando de los principios de este enfoque, y las empresas ya no asumen comportamientos optimizadores sino que eligen comportamientos satisfactores en función de un conjunto de opciones que contribuyen al logro de sus propósitos.

Coase postula que bajo costos de transacción nulos todos los agentes podrán buscar la información necesaria y realizar los arreglos contractuales requeridos para maximizar la producción, por lo que se lleva a cabo un intercambio eficiente entre las partes, 
independientemente de los derechos de propiedad. Este postulado es cercano a la lógica neoclásica, debido a que las transacciones de mercado bajo un sistema de precios eficiente permiten establecer la delimitación legal inicial de los derechos, que podrán reasignarse si esto conduce a un incremento del valor de la producción.

No obstante, este autor elimina el supuesto de nulidad en los costos de transacción, porque para realizar transacciones de mercado es necesario, entre otras cosas, definir con quién se desea efectuar el intercambio, informar sobre lo que se desea intercambiar y en qué términos, conducir las negociaciones que lleven a un convenio, redactar el contrato, así como llevar las acciones necesarias para asegurarse de que los términos del contrato se cumplan. Tales costos pueden ser de tal magnitud que pueden anular transacciones que tendrían cabida bajo un sistema de precios que funcionase sin costos.

Al margen de las transacciones de mercado, Coase apunta que existen otras dos posibles modalidades de organización de la producción representadas por la empresa y por la regulación del Gobierno. Dentro de la empresa individual se eliminan los convenios entre los distintos factores cooperantes en la producción y las transacciones de mercado se sustituyen por una decisión administrativa. El reordenamiento de la producción tiene lugar entonces sin necesidad de convenios entre los propietarios de los factores de la producción cuando los contratos son muy difíciles de lograr. La empresa representa, según el autor, una forma de organización productiva que podría lograr los mismos resultados a un menor costo. Esta solución se adoptaría cuando los costos administrativos de la empresa fueran menores que los costos de las transacciones de mercado que reemplaza, y las ganancias que resultarían del reordenamiento de las actividades fuesen mayores que los costos de organizarlas para las empresas. 
Por su parte, la regulación del Gobierno representa una solución alternativa. En vez de instituir un sistema legal que puede ser modificado por transacciones de mercado, el Gobierno puede imponer regulaciones que establezcan lo que la gente debe hacer o no, y cuáles deben ser obedecidas. Así, el Gobierno es capaz de influir en el uso de los factores de producción mediante leyes y organismos administrativos. Pero la máquina administrativa del Gobierno no es, en sí misma, sin costo. Para Coase (1960), por el contrario puede ser en ocasiones extremadamente costosa, por lo que deduce que la regulación gubernamental directa no necesariamente presentará mejores resultados que la solución de mercado o a través de la empresa, lo que demuestra la cercanía de este autor con el pensamiento neoclásico.

Otra de las vertientes neoinstitucionalista plantea un esquema donde la empresa opera en un ambiente con fallas de mercado y de racionalidad limitada. Williamson (1985) señala que los agentes económicos tratan de ser racionales, pero solo lo logran de forma limitada. Esta premisa se opone al enfoque neoclásico ortodoxo en el sentido de que: a) en el mundo real hay incertidumbre e información imperfecta; b) los agentes tienen capacidades de cálculo limitadas; y c) existe interdependencia estratégica, porque los resultados de las decisiones de cada agente dependen parcialmente de las decisiones que adopten los demás. Por tal motivo, las empresas no eligen la mejor opción posible sino la que creen que es mejor, dadas las limitadas capacidades de cálculo y de información que poseen. Lo que significa, en otros términos, que las empresas desarrollan comportamientos de «satisfacción» más que de «optimización».

Otro concepto central en Williamson es el de oportunismo, que repercute en los costos de transacción: los agentes no necesariamente respetan sus promesas o compromisos. Esto se refiere a la posibilidad que tienen dichos agentes de sacar provecho 
de nuevas situaciones durante el desarrollo de un contrato. En conjunto, tanto la racionalidad limitada como el oportunismo aumentan los costos y la complejidad del proceso de diseño, negociación y cumplimiento de los contratos. Lo que en otras palabras significa que elevan los costos de transacción.

Un tercer elemento en el análisis de Williamson se refiere a propiamente las transacciones, las cuales difieren por: a) la frecuencia con la que ocurren; b) el grado de incertidumbre al que están sujetas; c) la especificidad de los activos o el grado en que un activo puede ser dedicado a otro empleo sin perder valor. La frecuencia de las transacciones influye en el oportunismo, porque en la medida en que una transacción determinada se repite más frecuentemente resultan menos probables las conductas oportunistas. A su vez, las transacciones con alto grado de incertidumbre facilitan los comportamientos oportunistas.

Especial atención recibe la especificidad de los activos. Cuando hay transacciones de activos específicos la identidad de las partes contratantes cobra relevancia a causa de la mutua dependencia. Motivo por el cual se requieren otras formas de gobierno de las transacciones más allá de las que proporciona el mercado. Formas de gobierno que adquieren importancia y se vuelven más complejas a medida que se presenta, al mismo tiempo, una racionalidad limitada y actitudes oportunistas. Por ello, se tendrán diferentes formas de gobierno, dado que cada transacción tiene distintos atributos y, por tanto, diferentes costos de transacción.

No obstante, para autores como North (1990) los efectos de las instituciones no solo se reducen a los costos de transacción, sino que su función principal es reducir la incertidumbre entre los actores sociales, porque proporcionan una estructura que define y limita el conjunto de elecciones de los individuos. Por ello, los empresarios toman en 
consideración el marco institucional en el que operan, ya que les permite reducir la incertidumbre y tratar de minimizar los costos que implica la creación de empresas. Sin embargo, cada ambiente proporcionará un esquema institucional diferente con reglas formales y no formales, pautas culturales y cognitivas que introducen cierto grado de incertidumbre y de costos de transacción, lo que estimula o entorpece la empresarialidad.

Como se mencionó, las instituciones pueden ser tanto formales como informales. En el primer caso, y refiriéndose ahora en concreto al problema de la empresarialidad, las normas escritas así como su estabilidad y coherencia, formas de implementación y grados de aplicación, son un aspecto central que hará más o menos atractivo desarrollar actividades empresariales, e influirá sobre las formas y orientaciones que asuman dichas actividades. Lo mismo ocurre con las instituciones informales, porque los valores, creencias y convenciones, también operan sobre esos mismos fenómenos, aunque a través de canales diferentes y en muchas ocasiones resulten más complejos de entender y precisar.

Los valores, creencias y convenciones, juegan un papel fundamental en la formación de expectativas, esto debido a que en el corto plazo definen la forma en que los individuos procesan y utilizan la información. Por tal motivo, las instituciones no formales resuelven los problemas de coordinación y pueden producir comportamientos cooperativos. Pero los componentes de las instituciones informales también están en trasformación permanente, se modifican a partir de la experiencia y se filtran a las construcciones mentales existentes. Por ello hay un proceso continuo de formación de expectativas y de mecanismos de coordinación.

A partir de la incorporación del marco institucional al análisis económico, el concepto de equilibrio único de la teoría neoclásica ortodoxa se trasforma en diversos equilibrios, porque como señala North (1990) no se registra únicamente un solo equilibrio 
sino más bien se tiene un conjunto de equilibrios. Estos equilibrios son consecuencia de que los actores enfrentan diversas elecciones únicas y no repetitivas en las cuales la información es incompleta y los resultados inciertos.

Sobre estas bases se argumenta que las organizaciones y los agentes económicos intentarán adquirir la clase de habilidades y conocimientos que les permitan sobrevivir y prosperar en el marco institucional vigente, pero también tratarán, según los casos, de mantener o cambiar las reglas con el fin de aumentar los beneficios que reciben. Esto va en línea con el cambio institucional propuesto por North (1990), en donde se ve una influencia bilateral entre el comportamiento de los agentes y la estructura institucional.

Los emprendimientos tendrán efectos sociales diferenciados, según sea si se dirigen a actividades productivas, como por ejemplo la innovación, o a actividades improductivas buscadoras de rentas. A su vez, dicha decisión depende del esquema social de incentivos, que determina las retribuciones a obtener en cada actividad. Para este autor lo que cambia de un periodo o de un país a otro y define el efecto último sobre la economía es el conjunto de reglas y no tan solo la cantidad de emprendedores o la naturaleza de sus objetivos. A su vez, este marco institucional es el que facilitará u obstaculizará la creación de nuevas empresas.

\section{ENFOQUE EVOLUCIONISTA}

Este punto de vista, según Nelson y Winter (1982), y Dosi y Nelson (1994), tiene dos características esenciales: primera, su propósito es explicar un proceso a través del tiempo, lo que en otras palabras significa por qué ese proceso tiene ciertas características en un determinado tiempo y cómo es que llegó a tener dichas características; segunda, las 
explicaciones incluyen factores aleatorios que generan algunos cambios en las variables de análisis, y mecanismos que sistemáticamente reducen o extienden la variación.

Algunos de los elementos centrales del enfoque evolucionista son:

1. La permanencia — herencia- y mecanismos de trasmisión. Representan las rutinas o patrones de conducta regular y predecible que aplican los agentes económicos y sobre los cuales estos basan sus comportamientos cotidianos.

2. El principio de variación o mutación. La variación es producto de la introducción de manera exógena y endógena de «novedades» en el sistema económico. Dichas novedades representan nuevas firmas, tecnologías, modelos organizacionales, formas de gobierno corporativo, etcétera. También son generadoras de la «variedad» que alimenta las trasformaciones de los sistemas en el largo plazo y, por tanto, juegan un papel central en los procesos de desarrollo y cambio estructural. Parte de la dinámica es endógena al sistema en tanto que las novedades son, en gran medida, producto del propio aprendizaje de las firmas y de comportamientos de búsqueda, provocados por los cambios que enfrenta el individuo o la firma derivado del propio proceso de competencia en el mercado.

3. El mecanismo de selección que actúa sobre las rutinas y las mutaciones. El mecanismo selectivo incluye como actor central al mercado, pero también incorpora la influencia de otros actores y elementos como las políticas públicas, grupos de presión, tendencias macro económicas, etcétera. Dicho mecanismo actúa sobre las firmas, las tecnologías y las rutinas, seleccionando las conductas y agentes mejor adaptados al ambiente. 
Estos factores característicos del enfoque evolucionista representan un rompimiento con la visión neoclásica ortodoxa de los procesos económicos, aunque recuperan algunos de los elementos de la teoría neoinstitucionalista: la reducción de costos de transacción y la limitación de la incertidumbre entre los actores, factores que son incorporados en el enfoque evolucionista al expresar que existen mecanismos de permanencia que establecen patrones de conductas regulares y predecibles; así como por el mecanismo selectivo en que intervienen el mercado y otros actores sociales.

En el enfoque evolucionista se destaca que las empresas son heterogéneas y se caracterizan por diversas competencias, creencias y expectativas, de igual manera por diferentes grados de acceso y capacidad de procesamiento de los flujos de información. Más aún, Dosi y Nelson (1994) señalan que no solo las firmas son distintas, sino que además esas diferencias son importantes tanto en el ámbito micro como macro económico. Lo cual resulta clave para el avance del sistema como un todo, en tanto asegura que emerja una variedad de comportamientos más amplia y, por tanto, que exista un mayor número de fuentes de conocimiento e innovaciones, lo que favorece la eficiencia del sistema a largo plazo.

También se argumenta que las firmas operan en condiciones de incertidumbre y, de esta manera, no es posible que puedan emplear procesos de decisión basados en la maximización de alguna variable. En consecuencia, en este enfoque las firmas son consideradas como agentes heterogéneos y de racionalidad limitada que actúan en ambientes inciertos y complejos, aplicando rutinas que cambian solo lentamente a lo largo del tiempo. Las rutinas pueden ser entendidas como comportamientos que se estiman apropiados y efectivos para los contextos en donde son empleadas. En otras palabras, representan lo que mejor hace y puede hacer la empresa ante una determinada situación. 
Los cambios en las empresas tienen, en parte, un origen endógeno basado en los procesos de aprendizaje que llevan a la empresa a modificar sus estrategias y acumular competencias. Sin embargo, las empresas también reaccionan frente a las trasformaciones que ocurren en el ambiente. Lo que una firma puede hacer y las decisiones que puede adoptar están condicionados en gran medida por sus capacidades y experiencia previa. Esto significa que la evolución de las empresas en parte está determinada por su proceso de aprendizaje y en parte por las condiciones del contexto donde opera.

Las empresas, al no estar completamente determinadas por el marco en el que ellas actúan, ante un mismo contexto institucional y económico, e incluso un mismo sector, pueden adoptar estrategias y decisiones diferentes en función de sus objetivos, estructuras y capacidades. En consecuencia, en cada momento del tiempo habrá una pluralidad de estrategias empresariales que conviven y compiten en el mercado.

\section{EL DESARROLLO ECONÓMICO LOCAL}

Según Vázquez (1999), el desarrollo económico local ${ }^{4}$ implica un proceso de crecimiento económico y cambio estructural, impulsado por la comunidad local, que utiliza los recursos disponibles en el territorio y conduce a la mejora de las condiciones de vida de la población local. En este proceso lo social se integra con lo económico, porque los actores públicos y privados toman decisiones de inversión orientadas a aumentar la productividad y competitividad de las empresas, resolver los problemas locales y mejorar el bienestar de la sociedad.

De acuerdo con este autor se pueden identificar cuatro grandes dimensiones del desarrollo económico local: 1) la dimensión económica, compuesta por un sistema específico de producción que posibilita a los empresarios locales usar eficientemente los 
factores productivos y alcanzar los niveles de productividad que les permite ser competitivos; 2) la dimensión sociocultural, en la cual los actores económicos y sociales se integran con las instituciones locales formando un sistema de relaciones que incorporan los valores de la sociedad en el proceso de desarrollo; 3) la dimensión política, que se instrumenta mediante las iniciativas locales y que permite crear un ambiente local que estimula la producción y favorece el desarrollo sostenible; 4) la dimensión territorial, no tan solo por los efectos espaciales de los procesos organizativos y tecnológicos sino también por el hecho de que cada localidad o región es el resultado de una historia en la que se ha ido configurando el entorno institucional, económico y organizativo (Vázquez 1999).

Para la perspectiva del desarrollo económico local, cada espacio económico registra una configuración específica que se define en función de los sucesivos sistemas productivos, de los cambios tecnológicos y organizativos de las empresas e instituciones, y de las trasformaciones en el sistema productivo. A este respecto Johannisson (2006) señala que la organización territorial generalmente se identifica con el concepto de red, entre otros motivos porque:

1. Representa una forma de organización que se encuentra estrechamente asociada con la toma de conciencia de la importancia de los compromisos sociales mutuos entre los actores económicos. La identificación social resulta más intensa en contextos físicos cercanos donde se comparten cotidianamente escenarios mentales, sociales y territoriales.

2. Las formas en que se articulan las empresas en un territorio, donde se manifiestan relaciones de competencia y cooperación, adquieren características muy particulares según la historia de cada territorio. 
3. La localidad ofrece un escenario para la organización en la que participan el sector público, el sector privado y organismos no gubernamentales. Además de que los empresarios no responden únicamente a motivaciones de tipo económico, sino que también lo hacen por el reconocimiento social del medio en el que operan.

4. Se puede identificar la actividad emprendedora como un fenómeno relacional y colectivo que adquiere una connotación particular en cada territorio específico.

A partir de estos elementos se podría decir que la red representa una metáfora del modo en que son creados los procesos empresariales que afecta de manera específica el nacimiento de nuevas empresas. Las redes desempeñan un papel central en los procesos de desarrollo económico porque condicionan el surgimiento y crecimiento de las empresas, la difusión del conocimiento y de la innovación, así como el propio proceso de acumulación de capital.

La emergencia de empresarios, y de manera específica de pequeñas empresas, se apoya en la presencia de redes personales que permiten madurar los proyectos de empresa a partir de información que obtiene de otros agentes, de igual manera permite incluir a personas de su confianza, a las cuales conocían antes de que la idea surgiera y se trasformara en proyecto.

El contexto local, de acuerdo con Vázquez (2005), proporciona los recursos y las relaciones necesarias para el desarrollo de la actividad productiva. Además contribuye a reducir el grado de incertidumbre que se asocia con la actividad productiva, y proporciona a los empresarios la autoestima que necesitan para asumir los riesgos que implican los proyectos empresariales. También, los contextos económico, social e institucional, proporcionan a las empresas elementos necesarios para identificar y aprovechar las oportunidades que se presentan en los mercados. 
El territorio desempeña un papel central en el desarrollo empresarial, porque como apunta Malecki (1999) existe una gran variedad de factores culturales, sociales, institucionales y económicos, que interactúan entre sí y se refuerzan mutuamente; por lo que las características específicas del territorio son las que promueven el surgimiento y desarrollo de las empresas. Este enfoque emerge como contraposición a la visión tradicional del enfoque neoclásico ortodoxo que refiere a los empresarios como empresas que compiten entre sí y que se relacionan a través de las señales del mercado — precios y costos_ - en la búsqueda de beneficio. El surgimiento y crecimiento de la capacidad empresarial y organizativa en un territorio es el resultado de un proceso complejo donde las instituciones, las redes de contactos personales y las acciones estratégicas de los actores, permiten a los empresarios realizar su proyecto de negocio.

Como se puede observar, el enfoque de desarrollo local incorpora algunos elementos del enfoque institucionalista y otros del evolucionista. Los cuales sintetiza e incluye en el papel que desempeña el territorio como una configuración particular a partir de las características de tipo cultural, social, político y económico, que condicionan los procesos de empresarialidad local.

Dadas esas características específicas de la empresarialidad local, en la siguiente sección se comentarán algunas de las limitantes del modelo Global Entrepreneurship Monitor, GEM, para analizar la empresarialidad desde la perspectiva del desarrollo local, ya que uno de los objetivos de este modelo es incorporar variables que permitan captar las especificidades culturales y sociales que son propias de los análisis del desarrollo local. 


\section{EL MODELO GEM DE CREACIÓN DE EMPRESAS}

Según el modelo GEM todos los empresarios actúan con base en dos situaciones puntuales: por necesidad y por oportunidad. La necesidad se asocia más con aquellos individuos que están desempleados o subempleados, sus ingresos son insuficientes, tienen poca estabilidad en su puesto laboral e, incluso, se sienten poco realizados en el empleo que llevan a cabo. En el segundo caso, la oportunidad se da cuando se cree que se tiene un proyecto innovador para un bien o servicio, se visualiza una ventaja competitiva para ampliar la presencia en el mercado actual o futuro, o bien se cuenta con recursos propios para independizarse.

Para la construcción de los indicadores del modelo GEM se consideran cinco factores que denominan endógenos y que caracterizan al empresario actual o potencial. Factores que son: a) motivación; b) perfil; c) fuentes de financiamiento; d) tecnología empleada por el empresario; e) grado de innovación. Además nueve factores denominados exógenos: 1. normas culturales y sociales; 2 . educación y capacitación; 3 . financiación; 4. infraestructura física; 5. infraestructura comercial y legal; 6. apertura del mercado; 7. trasferencia de la investigación y desarrollo; 8. políticas gubernamentales; 9. programas gubernamentales.

El modelo GEM proporciona un instrumento que permite estudiar la empresarialidad en el contexto global de cada país, y al utilizar los mismos indicadores permite la comparación entre países; no obstante, esta metodología resulta insuficiente para conocer el comportamiento de la empresarialidad desde una perspectiva del desarrollo económico local. Para apuntar de manera específica algunas deficiencias del posible uso del modelo GEM en el análisis de la empresarialidad desde el ámbito local se comentan brevemente algunos de los factores endógenos y exógenos. 
Dentro de los factores endógenos, la motivación para emprender puede ser producto de aprovechar la oportunidad para realizar algún emprendimiento a partir de la idea específica de un negocio o bien el deseo de iniciar uno nuevo. También se considera la expectativa de incrementar los ingresos, o porque proporciona un medio para el autoempleo. En cuanto a la tecnología empleada por el empresario, esta variable se enfoca al tiempo en el cual el empresario puede acceder a la tecnología necesaria para producir el producto o servicio. Mientras que la variable de innovación considera a los clientes potenciales que pueden tomar en cuenta el producto innovador, así como a las otras empresas que ofrecen productos similares a los productos potenciales (Ibarra y Gómez 2007).

Si bien no se puede dejar de reconocer que en las motivaciones para realizar nuevos emprendimientos pueden estar presentes factores que se circunscriben al ámbito de la oportunidad o necesidad, sin embargo estos factores se moldean por las condiciones específicas que tiene cada territorio y no tan solo por el ámbito personal de los empresarios.

Por su parte, la tecnología y la innovación tecnológica no son productos totalmente acabados que se encuentren disponibles para que los empresarios hagan uso de ellos de manera eficiente, sino que la tecnología y la innovación requieren de procesos de aprendizaje y retroalimentación, que frecuentemente se realizan con otros agentes localizados en el territorio.

Dentro de los factores exógenos del modelo GEM destaca el referido a las normas culturales y sociales. Esta variable analiza la actitud general de cada persona hacia la creación de empresas, las actitudes de los ciudadanos hacia el nivel de riesgo que están dispuestos a asumir, el miedo al fracaso empresarial, las necesidades de creación de riqueza y su repercusión en el desarrollo empresarial. Desde la óptica social se analiza cómo las 
normas sociales impactan en los comportamientos de los empresarios y cómo la sociedad los valora (Ibarra y Gómez 2007). El factor cultural y social del modelo GEM incluye algunos de los aspectos importantes para identificar la influencia de estos factores en la creación de empresas. No obstante, es conveniente incorporar la parte de reglas, normas y valores no formales, sobre los cuales descansan muchas de las redes de cooperación que se constituyen en el territorio y que contribuyen a la empresarialidad local.

Las empresas representan un conjunto heterogéneo que exhibe conductas, estrategias y desempeños altamente diversos. Cualquier generalización o intento de homogeneización de su tratamiento implica desconocer esta diversidad. A su vez, poner a la diversidad en primer plano no solo es necesario para comprender mejor la dinámica micro económica sino también para analizar los procesos de desarrollo en el largo plazo. Esto es así en tanto conlleva la heterogeneidad de conductas y estrategias, mismas que garantizan que exista una verdadera competencia de ideas e innovaciones en la sociedad, factor crucial para el crecimiento a largo plazo. En otras palabras, si no incorporamos la heterogeneidad y diversidad micro económica al análisis, difícilmente podemos comprender la dinámica de conjunto de un sistema económico.

Más allá de que existan características personales que hagan a los individuos más o menos inclinados o aptos para convertirse en empresarios, el marco institucional y macro económico vigente en la sociedad tendrá una influencia decisiva sobre el tipo de actividades en las cuales ellos aplicarán sus capacidades y competencias, e incluso influirá sobre el tipo de competencias que los empresarios buscan desarrollar. Aquellas actividades pueden ser productivas o improductivas, innovadoras o especuladoras, con visión de corto plazo o comprometidas con el largo plazo, según el esquema de retribuciones vigente en cada caso y de la existencia de un esquema de proyecto impulsado desde los actores del 
territorio. En otras palabras, no hay sociedades con empresarios natos o genéticamente innovadores y otras sociedades donde predominan los no innovadores, sino que son los contextos territoriales los que definen el tipo de conductas y estrategias que serán apoyadas u obstaculizadas.

La relación entre empresas, instituciones y desarrollo económico, es cambiante a lo largo del tiempo. Las instituciones también se modifican, nacen o desaparecen, tanto por la acción conciente de la sociedad a través de la acción del Estado como por la evolución más o menos espontánea de las normas culturales, sociales, etcétera. Así, por ejemplo, marcos institucionales que en determinado momento inducen a los empresarios a comportarse de manera innovadora, en otros pueden favorecer comportamientos poco innovadores. Si bien las condiciones del territorio son fundamentales para comprender el comportamiento de los empresarios y en específico del nacimiento de nuevas empresas, no se debe dejar de lado que las trasformaciones en los contexto macro económico e internacional en los cuales se desenvuelven las empresas también influyen sobre los resultados de los emprendimientos.

En el caso de los países en desarrollo pueden darse «círculos viciosos» de estancamiento cuando se combina una macro economía volátil con un ambiente institucional que favorece la búsqueda de rentas en actividades improductivas. El subdesarrollo financiero y la presencia de altos costos de transacción suelen acompañar a este tipo de escenario. En dicho caso, el crecimiento a largo plazo se perjudica tanto por la ausencia de inversiones e innovaciones y por la dificultad para el desarrollo de sectores intensivos en conocimiento y de rápido ritmo de cambio tecnológico, como por la orientación de los agentes hacia la consecución de capacidades que no contribuyen a los objetivos de desarrollo económico y social. Aun cuando esta situación llegue a ser evidente, los cambios necesarios pueden resultar muy difíciles, en tanto que aquellos agentes que se 
benefician con las rentas especulativas e improductivas sean capaces de bloquear los intentos de reforma.

\section{CONCLUSIONES}

Partiendo de la idea de que la empresarialidad es un factor de suma importancia en el desarrollo económico local, se han revisado brevemente los enfoques neoclásico, institucionalista, evolucionista y de desarrollo económico local. De dicha revisión se desprende que los empresarios no se dedican a efectuar los cálculos que les permitan la maximización de los beneficios en función de la aplicación eficiente de los factores productivos según se lo indiquen las señales del mercado.

Por el contrario, los empresarios se desenvuelven en un contexto institucional con reglas formales y no formales, así como con ciertos patrones culturales que proporcionan certidumbre pero que a su vez generan costos de transacción. Aunado a los esquemas institucionales, se reconoce que las empresas son heterogéneas, con una diversidad de competencias, creencias y expectativas, y que por lo tanto registran una pluralidad de estrategias. Las decisiones de las empresas, en parte tienen un origen endógeno determinado por su experiencia previa y la capacidad de aprendizaje. Por otra parte, las empresas también modifican sus decisiones en función de las trasformaciones que se generan en el contexto macro económico y en el ambiente donde operan.

Incorporar la dimensión territorial permite visualizar la actividad emprendedora como un fenómeno relacional y colectivo que registra especificaciones particulares según sea el territorio de que se trate, porque cada localidad es resultado de una configuración cultural, institucional, económica y organizativa. La perspectiva del desarrollo local indica, por tanto, que la empresarialidad responde en parte a las pautas organizativas del sector 
privado, del sector público y de organismos no gubernamentales. Tales pautas organizativas pueden responder a la presencia de fuertes compromisos sociales que se manifiestan a través de las redes personales que pueden servir como sustento al surgimiento de nuevas empresas.

Finalmente se menciona que para el análisis del desarrollo económico local los instrumentos generales de análisis de la empresarialidad, como el modelo GEM, no incorporan el ámbito territorial ni los factores que moldean las condiciones de necesidad y oportunidad. Tampoco se consideran en el modelo algunos de los elementos de las reglas, normas y valores no formales que son específicos de cada territorio y que condicionan el comportamiento de la empresarialidad. 


\section{BIBLIOGRAFÍA}

Acs, Z. y D. Audretsch, 2005, «Entrepreneurship, innovation and technological change». En The paper on entrepreneurship, growth and public policy, editado por Group entrepreneurship, growth and public policy. Max Planck of Economics, Jena.

Acs, Z. et al., 2005, «Global entrepreneurship report. 2004 executive report». Babson College-London Business School, London.

Audrestch, D., 2002, «The dynamic role of small firms: Evidence from the U.S.». Small Business Economics, vol. 18, núms. 1-3, pp. 13-40.

Audrestch, D. y R. Thurik, 2001, «Linking entrepreneurship to growth». STI Working Paper, núm. 2. OCDE.

Bates, T., 1993, «Theories of entrepreneurship». En Theories of local economic development. Perspectives from across disciplines, pp. 248-264, editado por Bingham, R. D. y R. Mier. Sage, Newbury Park, London, New Deli.

Coase, R. 1960, «The problem of social cost». Journal of Law and Economics, vol. 3, pp. 144.

Cohendet, P. y P. Llerena, 1998, «Theory of the firm in an evolutionary perspective: A critical development». Paper to the conference Competence, Governance and Entrepreneurship, 11-13 de junio. Copenhagen.

Coriat, B. y O. Weinstein, 2002, «Organizations, firms and institutions in the generation of innovation». Research Policy, vol. 31, pp. 273-290.

2005, «La construcción social de mercados». Noticias de la regulación, núm. 53. 
Dosi, G. y R. Nelson, 1994, «An introduction to evolutionary theories in economics». Journal of Evolutionary Economics, vol. 4, núm. 3, pp. 153-172.

Friedman, R., 1988, «Entrepreneurial renewal in the industrial city». Annals of American Academy of Political and Social Science, núm. 488, pp. 35-46.

Grebel, T. 2004, Entrepreneurship. A new perspective. Routledge, London.

Hall, R. y C. Jones, 1999, «Why do some countries produce so much more output than others?». Quarterly Journal of Economics, pp. 83-116.

Hallberg, K., 2000, «A market oriented-strategy for small and medium scale enterprises». Discussion paper, núm. 40. World Bank-International Finance Corporation, Washington, D.C.

Ibarra, A. y L. Gómez, 2007, «Hacia un diagnóstico latinoamericano para la creación de empresas con la aplicación del modelo GEM 2006». Pensamiento y Gestión, núm. 22, pp. 85-142.

Jacobsohn, G., 2004, «La creación de nuevas firmas: Aportes de la dirección estratégica». Pensamiento y Gestión, núm. 16, pp. 135-157.

Johannisson, B., 2006, «El desarrollo empresarial mediante la red de contactos empresariales». Ekonomíaz, núm. 62, 2. ${ }^{\circ}$ cuatrimestre, pp. 220-239.

Lucas, R., 1990, «Why Doesn’t Capital Flow from Rich to Poor Countries?». American Economic Review, Papers and Proceeding, vol. 80, núm. 2, pp. 97-96.

Malecki, E., 1999, Technology and economic development: The dynamics of local, regional and national competitiveness. Longman, London. 
Nelson, R., 1991, «Why do firms differ, and how does it matter?». Strategic Management Journal, vol. 12.

Nelson, R. y S. Winter, 1982, An evolutionary theory of economic change. Belknap Press, Cambridge.

North, D., 1990, Institutions, institutional change and economic performance. Cambridge University Press, Cambridge.

Organización para la Cooperación y el Desarrollo Económico, OCDE, 1997, «Small businesses, job creation, and growth: Facts, obstacles, and best practices». En http://www.oecd.org/dataoecd/10/59/2090740.pdf. Paris.

2001, «Entrepreneurship, growth and policy». Paris.

Porter, M., 1998, «The competitive advantage of inner city». En On competition, pp. 377408, editado por M. Porter. Harvard Business School Publishing, Boston.

Reynolds, P. et al., 2001, 2002, «Global Entrepreurship Monitor, Executive Reports». En www.entreworld.org

Vázquez, A., 1999, Desarrollo, redes e innovación. Lecciones sobre desarrollo endógeno. Ediciones Pirámide, Madrid.

2005, Las nuevas fuerzas del desarrollo. Antoni Bosch, Barcelona.

Williamson, O., 1985, «The Economic Institutions of Capitalism: Firms, Markets, Relational Contracting». The Free Press, New York. 


\section{Notas}

${ }^{1}$ Por enfoque neoclásico convencional se entiende la teoría neoclásica ortodoxa, que asume los supuestos de competencia perfecta, perfecta información de mercados, libre movilidad de recursos, perfecta sustitución de factores, etcétera.

${ }^{2}$ El proyecto Global Entreprenurship Monitor nació en el año 1998 como propuesta de London Business School y de Babson Collage como una metodología general para estudiar la empresarialidad.

${ }^{3}$ No todo el análisis neoclásico tiene una concepción de empresario tan limitada como en la escuela marginalista. En el trabajo de Grebel (2004), se puede entrar en un análisis de la concepción de empresario dentro de la escuela neoclásica y de otras escuelas del pensamiento económico.

${ }^{4}$ Antonio Vázquez (1999) para referirse al desarrollo económico local utiliza como equivalente el término de desarrollo endógeno.

Fecha de recepción: 22 de febrero de 2008

Fecha de aceptación: 25 de abril de 2008 